\title{
Reliability of Human Subject - Artificial System Interactions
}

\author{
M. Novák, J. Faber, Z. Votruba, V. Přenosil, T. Tichý, P. Svoboda, V. Tatarinov
}

Main problems related to reliability of interaction between human subject and artificial system (namely of the transportation character) are discussed. The paper consists of three mayor parts:

The first one is devoted to the theoretical backgrounds of the problem from the both theory of system reliability and neurology/psychology views.

Second part presents the discussion of relevant methodologies of the classification and prediction of the reliability decline. The methodology based on EEG pattern analysis is chosen as the appropriate one for the presented task. The key phenomenon of "micro-sleep" is discussed in detail.

The last part presents some latest experimental results in context of presented knowledge.

Proposals for the future studies are presented at the end of the presented article. The special interest should be devoted to the analysis and in-time prediction of fatal attention decreases and to the design and construction of the respective on-board applicable warning system.

Keywords: man-system interaction, reliability, attention, vigilance, classification, prediction, EEG analysis, micro-sleep, neuroinformatics.

\section{Introduction}

None of the many artificial systems, that human society has daily deals with on a daily basis can operate entirely independently - all of them still have to be controlled, or at least supervised, by man.

We will not here study the problem of whether or when (maybe sometime in distant future) artificial systems can be developed that will be fully independent on human beings. As far as we can see, this seems not to be probable. All the artificial systems that we need for our life, now and in the foreseeable future, will require interaction with a human operator (see e.g. [18]).

The quality and reliability of such an interaction is therefore of extreme importance for all of us, irrespective of the level of intelligence of the particular artificial system.

\section{An overview of the current state-of the art}

Very long experience of human beings dealing with artificial systems leads to the regrettable observation, that the human factor is very often the weakest point in the interaction.

The reason for this is quite natural and easy to understand:

Unlike artificial systems, humans cannot operate indefinitely without a break - human beings need to relax, rest and sleep. Decreasing human vigilance and attention while operation or using a given system has always been and still is the most frequent cause of system failures and accidents.

Another source of system failures lies in the possibility that the human operator (or user) of a particular artificial system may react too late, and that his/her decision and reaction may be incorrect.

Human behavior is not fully deterministic; it varies from subject to subject.
All these factors combine, with the result that the reliability of human subject - artificial system interaction is limited, above all from the human side.

The price that we all pay for insufficiently reliable human - artificial system interaction (above all from the human side) is tremendous.

Moreover, this price increases with time and also - surprisingly - with the level of the sophistication of the artificial system.

We urgently need to counteract this human unreliability.

Various approaches can be taken in an attempt to minimize the hazard of system operation failures. However, the following are main ways of improving system reliability (see [4]):

- by making the system as robust as possible, preferring to use of highly reliable (and therefore massive and expensive) components for its construction.

- by duplication and often even multiplying the most important parts of the system, or even duplicating or multiplying whole systems, which may operate as "hot" reserves. Like the first approach, this can also require very great effort and expense.

- a more sophisticated approach tries to modify the system structure in a way that minimizes the sensitivity of its system functions to parameter changes.

- in very sophisticated systems the predictive diagnostic approach can be applied, which enables us to analyze how much and in what direction the values of some system parameters can deviate from their nominal values without affecting the properties of the system as a whole, and to predict with what probability and when these limits of acceptable system behavior will be broken.

These four main approaches are in practice usually combined.

However, as concerns the reliability of interactions between human subjects and artificial systems, only the last approach seems truly able to minimize the frequency of system operation failures caused by human error and failure 
(see [23]). Of course, the first approach, which favors selecting well-qualified operators and then training and testing them, is also very important.

This paper on problems related to the technical aspects of reliability of interactions between human subject - either in the role of an operator (driver, pilot, dispatcher), supervisor or user - and an artificial, namely technical (and above all transportation) system, therefore concentrates on creating a practically applicable tool for diminishing the losses incurred when such interaction reliability is diminished.

The problem of interaction reliability between artificial systems and human beings (operators, users), though extremely important, has not yet been solved satisfactorily, even though most of complex and complicated systems are now controlled by computers or with the aid of computers (see [1, $2,3,5,6,7,10,11,12,16,17,22,23])$. A human operator, who has to interact with a powerful, complicated and often-efficient artificial system (e.g. a transport system, aircraft, express train, large truck, extensive power station, security or defense systems, etc.) needs to react rapidly and correctly to very variable situations over a long period of time.

The high load on the brain and nervous system of the operator results in an inevitable decrease in his/her vigilance, and in loss of attention.

The complex demands on a human operator interacting with a complicated and powerful artificial system are moreover often combined with the influence of some internal and external factors (such as long length of service, psychic isolation in the course of service, the current mental and physical state of the operator, climatic conditions, the quality of the environment in the given cockpit or control room, the monotony of the scene or image, that the operator has to observe, etc.). The psychic load of the system operator and the presence of pre-service or in-service stress factors can play a very important role in the reliability of a human subject - artificial system interaction.

We also have to take into account various other factors that are, typical for humans, namely:

- the extremely high variability of human subjects, especially as concerns intelligence, education, level of operation training and skill, ability to concentrate, tendency to irrational and panicky reactions to unexpected situations, general disposition, aggressiveness, etc.

- all human subjects are individuals, none react identically.

- human subjects dealing with artificial systems differ greatly in age, race and sex.

- unlike artificial systems, the reactions of human subjects cannot be exactly repeated - a human subject learns from experience and modifies his/her behavior to use a minimum of physical and psychic energy for controlling or applying the system.

All these factors seriously complicate the problem - we cannot use standard methods of control and system engineering. Our methodology needs to change from the standard psychologically - oriented approaches used for decades in system operator education and training to more modern approaches, based on the better knowledge of system reliability theory, mathematical analysis, artificial neural networks, numerical methods, etc.
This makes our problem much more complex - we need to combine knowledge from very different areas of science.

\section{Theoretical backgrounds of interaction reliability}

Artificial systems can interact with human beings on the basis of:

- human control of system operation,

- human supervision of system operation,

- human use of system operation,

- human society interaction with system operation.

All these four fundamental forms are important and in practice they are very often combined. For the first three forms, reliability and safety of system operation depends directly on the reliability and safety of these interactions.

In all these cases, interaction failures can lead to fatal situations, or at least to serious economic losses.

In the case of interaction between an artificial system and various members of human society forming its environment, interaction reliability and predictability can also be of very great importance. This is especially true in situations when the artificial system suddenly changes its behavior, and when it interacts with a large and heterogeneous part of human society. In order to estimate this environmental reaction, we need a deep understanding not only of the individual behavior of a human subject exposed to interaction with the varying properties of a particular artificial system, but also of any social factors that may exist or may be activated in the relevant part of human society. Such studies are evidently of great importance for general safety, but they are very difficult and time-consuming and require access to large special databases storing the results of many measurements of human subject interaction reliability markers. The Micro Sleep Base to be discussed below is an example of such a database.

As mentioned above, the unsatisfactory reliability of almost all-artificial systems used by man throughout history is a major problem. This is caused not by the low reliability and short lifetime of the artificial systems themselves, but by various errors by the human operators who deal with such systems. Recently, technological progress in the reliability of artificial systems has improved greatly. Consequently the probability of technical faults in well-designed and well-manufactured artificial systems is now usually very low (though unfortunately still not zero).

However, the probability of failure resulting from misuse and faults in system operator activities has increased rapidly. follows:

The main reasons for this unacceptable situation are as

- increasing complexity of the systems,

- increasing demands on the operator's ability,

- increasing demands on his/her level of continuous, long-term attention, and

- the increasing demands on the speed of his/her reactions.

The losses resulting from artificial system operation faults are proportional to their power, significance and value. In 
the case of many modern transportation systems (large planes, fast trains, large ships, trucks), large power plants, major financial systems, security and defense systems, and also major medical care systems, the losses resulting from a malfunction can be catastrophic.

Therefore, alongside the continuing interest in minimizing the probability of technical failures in any artificial system (at an acceptable cost), considerable interest has also been shown in recent years in the reliability of system operator activity. Studies demonstrate that human error accounts of the losses incurred by artificial system malfunctions.

The demands on a human operator of an artificial system can be categorized as follows:

a) demands on attention level and attention span,

b) demands on the speed of the operator's reactions,

c) demands on the correctness of the operator's decisions.

Let us suppose that these three above mentioned kinds of requirements can be expressed as the real numbers $x_{i}$ of some significant parameter $x_{\mathrm{i}}, i=1,2,3$ which expresses the level of attention, the speed of reaction and the correctness of the operator's decision. The acceptable values of the vector $\mathbf{X}=\left\{x_{1}, x_{2}, x_{3}\right\}$ fill out in space $\mathbf{X}$ some region $\mathbf{R}_{\mathbf{A}}$, called the region of acceptability. The concept of regions of acceptability is well known from the theory of tolerances of system parameters (see [4]). In the theoretical case that the parameters $x_{\mathrm{i}}$ are independent, the region $\mathbf{R}_{\mathbf{A}}$ has a rectangular shape. However, in practice, there are certain correlations between $x_{1}, x_{2}$ and $x_{3} . \mathbf{R}_{\mathbf{A}}$ therefore has a complicated shape.

The analysis of $\mathbf{R}_{\mathbf{A}}$ is a very important but usually rather laborious problem, for which many methods have been developed. Though the set of these methods is still not closed, for the purpose of this paper let us suppose that we know how to analyze the region of acceptability of interaction of operators with a certain system.

Let us also suppose, that we are dealing only with so called well operated systems, i.e., with operator - system interactions that work well, at least initially.

The following theorem can therefore be formulated:

Each real interaction between a human operator and an artificial system, when exposed to the influence of a set of independent variables $\mathbf{P}$ (time is usually - in dynamic systems - one of them, can be represented by some position of vector $\mathbf{X}$ in the parameter space, moving along a certain

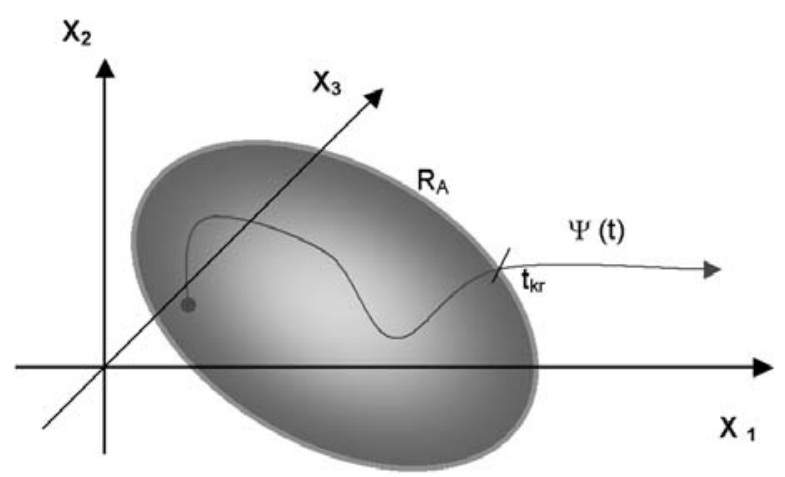

Fig. 1: An idealized run of the interaction life-curve of an operator - system interaction inside and outside the respective region of acceptability interaction life-curve $\Psi(t)$. For $t \rightarrow \infty$, the life-curve $\Psi(t)$ at least once breaks the boundaries of $\mathbf{R}_{\mathbf{A}}$.

This is illustrated in Fig. 1.

In accordance with the general theory of system reliability, we can state that:

The reliability of some operator - system interaction is represented as the probability $H$ that for a certain time interval (or some interval of another independent variable influencing the system under consideration) the respective interaction life-curve $\Psi(t)$ will remain inside the region of acceptability $\mathbf{R}_{\mathrm{A}}$.

Similarly,

The safety of the operator - system interaction is to be considered as the probability that it will be resistant to any disturbing influences.

A straightforward correlation exists especially between attention level and speed of reaction. Operators demonstrating a high level of attention usually also possess very fast reactions. On the other hand, cases can occur, when a fast, almost impulsive reaction may not be accompanied by very a high level of the operator's concentration and attention. Some people can react fast even, when their attention is dispersed on very different objects (they have very fast but unreliable reflexes).

In addition, a high level of attention leads in most cases to a very high probability of correct decisions and conversely, if somebody is not concentrating enough, there is a rather low probability that his/her decision will be correct.

On the other hand, in the case of very fast reactions accompanied by a very low level of the human operator's attention, the probability of an incorrect decision can increase significantly. This is typical for a so-called surprise reaction, which can sometimes change to a panicky reaction.

A drop in the attention level of a given human operator can be caused by various external or internal reasons; some of these have a general character; the intensity of others depends significantly on the operator's individuality.

General conditions causing decrease in attention include:

- extreme length of service without a break,

- physical and mental exhaustion,

- a monotonous scene, which the operator has to observe for a long time,

- extreme temperature in which the operator has to serve (too high or too low),

- extreme humidity in which the operator has to serve (too high or too low),

- extreme air pressure,

- air smell, dust density, etc.

Situations leading the operator to concentrate on problems other than his/her main task can likewise cause attention to drop. If the task is also monotonous, this can lead to a micro-sleep.

Of course, in practice, the shape of $\mathbf{R}_{\mathbf{A}}$ and $\Psi(t)$ is much more complicated, as shown in schematic Fig. 1.

For example occasionally the situation occurs that the operator's attention can improve in the course of his/her interaction with the system even if he/she was already in the 
stage of micro-sleep. In such a case, the respective interaction life-curve $\Psi(t)$ returns inside $\mathbf{R}_{\mathbf{A}}$, and after some time the procedure of attention decrease starts again. Such episodes, schematically expressed in Fig. 2 for two repetitions, can be repeated several times. In fortunate case the operator reaches the end of his/her service without an accident, or, in an unfortunate case, an accident occurs.

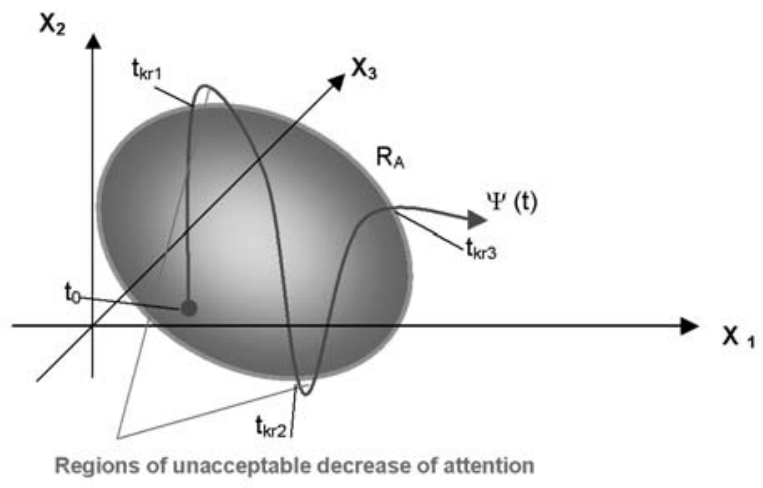

Fig.2: Repeated breaking of $\mathrm{R}_{\mathrm{A}}$ boundaries by $\Psi(t)$ in two temporary episodes and one final micro-sleep episode

The number of possible repetitions of such breaking of $\mathbf{R}_{\mathbf{A}}$ boundaries in micro-sleep episodes depends on many factors, including the individuality of the particular operator, his actual mental and physical state, the environmental and technical conditions of system operation and control etc. Though this is a very important problem, present-day knowledge is far from adequate, and much more research needs to be done in this area.

As in many cases when the regions of acceptability of technical system operation are investigated, here, too, the bound- aries of the regions of acceptable interaction are very often fuzzy.

\section{Human vigilance and attention classification and prediction}

Before starting a discussion of the problems of human subject attention decrease and micro-sleep, we will attempt to define this curious state of the human organism.

The available literature offers varied descriptions of this phenomenon. It is usually characterized as a state of an organism in which the eyes are closed and vigilance approaches zero. However, micro-sleep can also be understood as a state of the organism when its vigilance decreases below a certain limit, without reference to the activity of the visual tract. There are also several other conceptions of micro-sleep that lie between these two limits.

When dealing with man-system interaction reliability, decrease of vigilance seems to be much more significant than closing of the eyes, but a break in input of visual information longer than a certain limit ( e.g., about 1 second for car drivers) can also be dangerous.

The later mentioned definition has been considered for the purpose of $[24,25]$ to be a reasonable basis for the concept of micro-sleep, and will be used in further discussion:

First, however, we have to state what we mean by vigilance and what we mean by attention:

Vigilance... The state of the organism in which all its mental functions can be realized and when all receptor signals are accepted and well processed.

Attention... The form of vigilance, when the dominant part of mental functions is concentrated on external objects

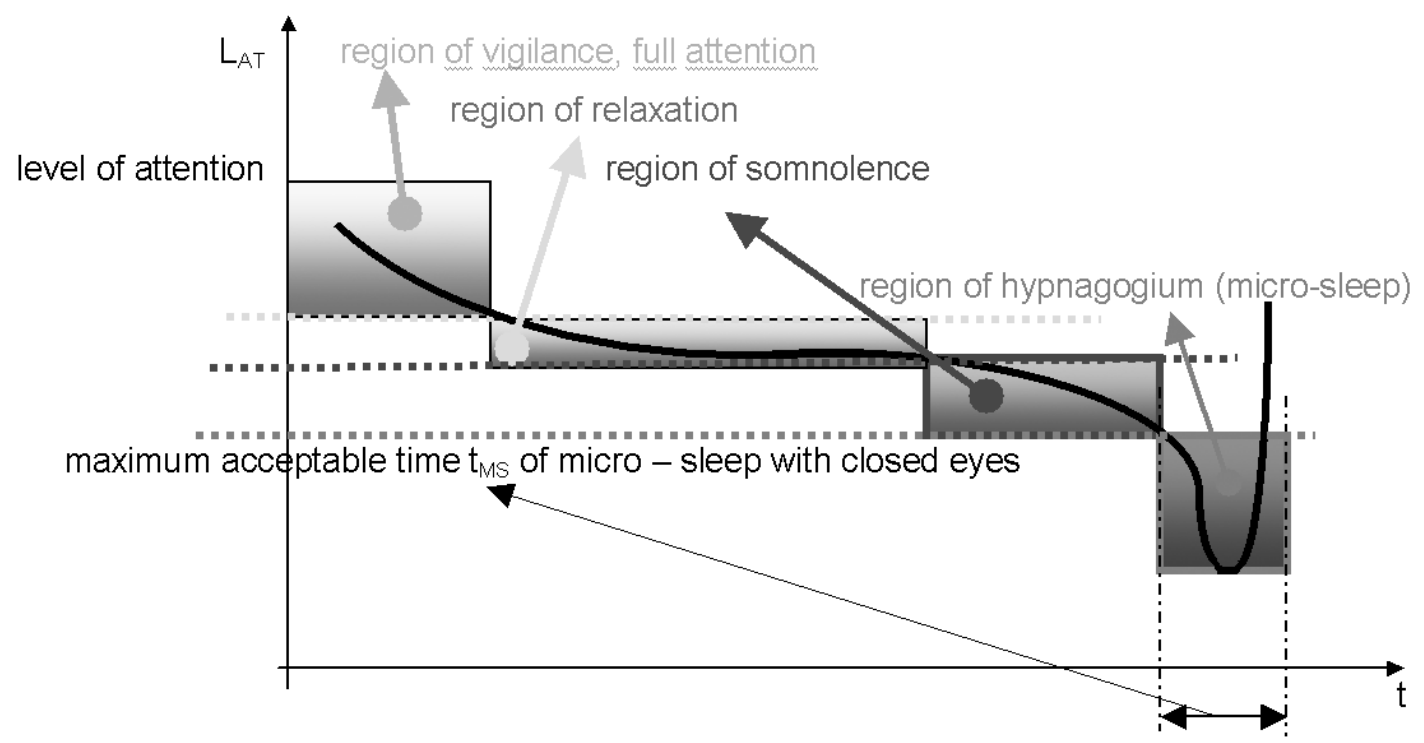

time $t$ in which the subject's mental activity $L_{A T}$ is observed

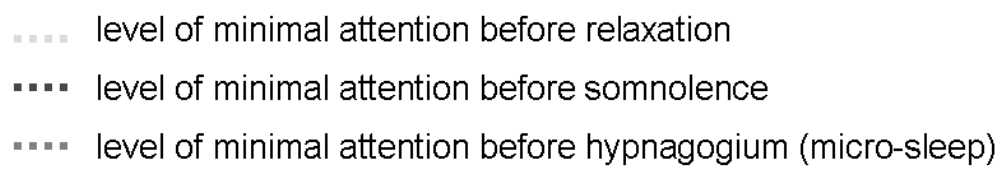

Fig. 3: Decrease of attention in the course of a human subject's activity 


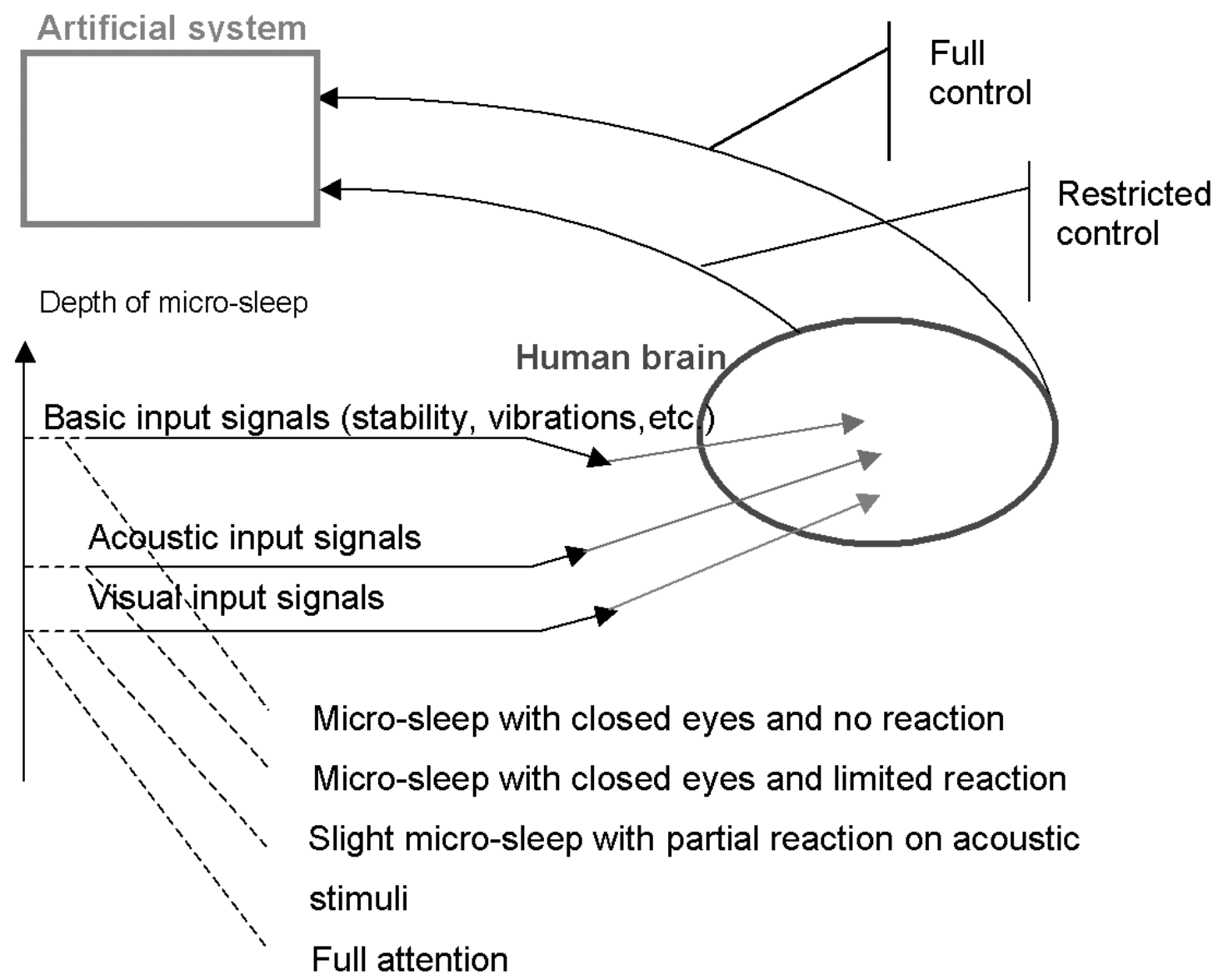

Fig. 4: Control functions in a human-system interaction during micro-sleep

(focused attention is considered as concentration on a certain object).

In a certain sense, attention can be considered as a special case of vigilance. In the state of vigilance, the human operator can react to all received signals. In the state of attention, she/he is usually concentrating on a certain group of received signals, which are dominant for the function she/he has to perform. In the state of attention, the sensitivity of the operator can be lowered to other non-dominant (secondary) signals. In further discussion of the problems of micro-sleep, we shall deal with the term "attention" only, because this state seems to be more characteristic for human operators who are able to interact well with artificial systems and control them properly.

Let us suppose that the level of a human operator's attention can be measured by the use of some real figure of merit $L_{\mathrm{AT}}$, expressed by real numbers. A discussion of some ways to express $L_{\mathrm{AT}}$ and to measure it will be presented later.

As is schematically shown in Fig. 3, the level of attention $L_{\mathrm{AT}}$ decreases with time over which the mental activity of the human subject in the course of his/her interaction with the artificial system is observed. Here we can distinguish the following four basic stages:

a) vigilance (full attention), in which the subject is completely competent to control (or use) the system under consideration,

b) relaxation, in which the subject is still competent to deal with the system under consideration, but where his/her at- tention decreases subsequently. This stage can last for a considerably period of time.

c) somnolence, in which the competence of the subject to interact with the system under consideration becomes restricted. This stage can also last for a considerably period of time, but unlike the previous stage, there is now a real danger that the subject will make control faults.

d) hypnagogium, in which the subject falls into a micro-sleep, at first with open eyes, but with very limited ability to control the system under consideration. He/she later falls into a micro-sleep with closed eyes, in which the competence of the subject to control the system is almost zero (some very skilled drivers - namely professional truck drivers - are able in this stage to hold the moving vehicle in a straight line, but they cannot react adequately to any curvature or barrier on the road).

In general, micro-sleep can be defined as follows:

Micro-sleep is a state of the human organism in which the mental vigilance and attention of the human operator controlling artificial system decreases below a certain limit.

This stage is very dangerous in both its phases. Fortunate subjects awake from this stage after a short time (especially from the phase of micro-sleep with closed eyes), usually quite rapidly. In the course of such an awakening the level of subject's attention increases rapidly, sometimes for a short period to a higher level than before the micro-sleep episode. However, in this short period of time there can be a higher 
probability of incorrect responses to stimuli, and there is, also a possibility of so-called panic reactions.

Micro-sleep episodes can be of various lengths. For each kind of human subject interaction with an artificial system there exist some limits of maximum acceptable decrease of attention and of length of micro-sleep, beyond which the given micro-sleep must be considered as dangerous.

This discussion should be supplemented by at least the following considerations:

a) the minimum acceptable level $L_{\mathrm{ATmin}}$ of the mental attention $L_{\mathrm{AT}}$ of the human organism depends significantly on the demands for a certain application of a human operator - artificial system interaction.

b) the micro-sleep episodes can be classified according to their general length in $t$ and according to the depths of the decrease in $L_{\mathrm{AT}}$ level (depth of micro-sleep).

The stages belonging to the class of micro-sleep with open eyes are usually a precursor of micro-sleep with closed eyes. In such a state of the organism certain level of vigilance still exists, but the subject's attention is considerably lowered and his/her reaction time RT is significantly prolonged. The probability of a correct and rapid decision on how to react to stimuli can decrease significantly.

This kind of micro-sleep is very dangerous. This stage can last for long period of time (and may take the form of a light or shallow micro-sleep) in which a given system operator is still able to control the respective system, though often only partially and insufficiently reliably.

After some time the light micro-sleep usually modifies into a real micro-sleep with closed eyes. This second class of micro-sleep usually shows some similarities to the regular REM phase of real night sleep but it lasts for a much shorter time. An operator falling into a micro-sleep with closed eyes cannot respond to any change of the system parameters, that he/she has is responsible for controlling.

Of course, there are exceptions. As was mentioned above, some professional truck drivers can fall into a special form of micro-sleep with closed eyes in which they are for some time still able to keep a moving truck in the right position on a straight road. Some very skilled drivers say that this can last up to several hundreds of seconds. The human control activity is here restricted to a small set of basic control functions, which are produced automatically without any higher feedback from input receptors (see Fig. 4).

We can suppose that driver in the stage of micro-sleep with open eyes can react to some input signals of an acoustic and visual character, though much more slowly and with greater probability of a wrong reaction. When he falls into this light form of micro-sleep with closed eyes, he may be able to react to some basic input signals of a mechanical character, such as vibrations, acceleration, deceleration and position (stability). In a very deep micro-sleep with closed eyes he/she cannot react at all.

Micro-sleep with open eyes can last a considerable time, and the operator's attention in such a situation is still near to the limit of the acceptable level. An operator sleeping in such a form of micro-sleep has changed (lowered) other significant parameters (markers) of his attention, and he/she is therefore scarcely able to be in control of any artificial system. This can be a dangerous situation.
The human operator's attention is represented in Fig. 3 by a scalar figure of merit $L_{\mathrm{AT}}$. In maximum simplification, this can be considered as the inverse of reaction time RT.

When the human subject, while operating an artificial system, falls into the stages of relaxation, somnolence and hypnagogium, his/her reaction times prolong significantly. While in full vigilance, the typical values of RT are about $200 \mathrm{~ms}$, for the stage of somnolence RT values in the range from $400 \mathrm{~ms}$ to $500 \mathrm{~ms}$ appear and in the stage of hypnagogium the RT values can exceed $800 \mathrm{~ms}$. If the human subject load is further prolonged, he/she usually falls into a real micro-sleep with closed eyes and his/her RT increases up to the time of awaking (this may be several minutes). RT values below $200 \mathrm{~ms}$ in full vigilance are found only in exceptional cases.

The level of danger caused by such prolongation of RT varies according the kind of human interaction with an artificial system. Typically, for car drivers, values of RT above $400 \mathrm{~ms}$ represent a distance of about $15 \mathrm{~m}$ at a speed of $100 \mathrm{~km} / \mathrm{s}$, over which the vehicle runs without any specific control (braking, turning etc.) corresponding to the stimulus (signal) received by the driver's sensors (to this distance we must, of course, add the distance, due to technical reasons, e.g., braking time).

Nevertheless, representing of $L_{\mathrm{AT}}$ by RT seems to be quite easy and physically transparent. However, for more accurate representation, a higher number $N_{\mathrm{AT}}$ of parameters $x_{\mathrm{i}}, i=1 \ldots N_{\mathrm{AT}}$ have to be taken into consideration. These parameters are called micro-sleep markers. An exact analysis of them can be quite laborious. For simplicity, in this paper we will deal with a reduced set of micro-sleep markers.

Our investigations at present are aimed at diagnosing attention decrease and the related reduction in speed of reaction to an unexpected situation. Such attention degradation can also lead to real micro-sleep. Both these critical types of states of the brain of an operator can be extremely dangerous and can result not only in huge material and financial damages, but also in loss of human life.

\section{Methodology of the study}

In order to detect a decrease in attention, we need to select a set of significant parameters which can be used for identifying attention decrease and the onset of micro-sleep.

Such parameters include:

- electro-magnetic activity of the brain,

- frequency of breath,

- frequency of heart beats,

- eye movements,

- skin resistance,

- facial grimaces, etc.

All these parameters have their specific advantages and also disadvantages.

We have chosen EEG activity as the dominant significant parameter, because this is probably the only parameter from which almost immediate and reliable information about brain function can be analyzed (similar information can be of course be obtained from magnetic measurements of brain activity, but this would be technically much more difficult). 
Analysis of EEG signals is a field of interest for many researchers specializing not only in neurology, but also in electrical control, signal engineering and mathematics (see $[8,9,12,14,15])$.

EEG measurements can be made quite well in laboratory conditions, and we have achieved very satisfactory results. Our preliminary results with EEG measurements in a moving vehicle are also quite promising. There is a real hope that in not distant future such EEG measurements can be performed in practical applications in moving vehicles or planes.

After investigating the information from many EEG time-series recorded on several dozen human subjects (probands) in our laboratory, we can analyze the process of the decrease in operator vigilance and attention degradation almost immediately, and with a quite acceptable level of reliability. As basis for this information mining we have used some relations that can be found between the components of an EEG signal.

Here we must of course take into account that the time-series representing the sampled EEG signal are in principle of a quasi-periodic and quasi-stationary character. Therefore, the classical concept of a spectrum for such time-series is not valid. The standard methods for spectral analysis, based on Fourier decomposition into the sum of periodical functions do not give accurate, representative and replicable results. Nevertheless, they continue to be widely used for this purpose, though the results are of limited use. To avoid any misunderstanding, we will denote such results below as pseudo-spectra. Nevertheless, careful analyses based on long-time experience of a skilled human expert enable much useful information and knowledge to be mined from the sets of data obtained by such pseudo-spectral analyses.

Notice: One serious drawback here is caused by the fact that in many neurological laboratories equipped with commercial EEG analyzers no information is made available about the kind of fast Fourier transform used in the particular measuring set, because the manufacturer treats this as his intellectual property. The results of such analyses, obtained in various laboratories, are therefore not fully compatible. To avoid this source of uncertainty, we have stimulated the development of fast pseudo-spectral analysis based on Gabor filtration, for which the respective polynomial filtration function of the $50^{\text {th }}$ order was designed.

However, there is good reason to hope that other non-spectral approaches to quasi-periodical and quasi stationary time-series will lead to the development of a more accurate and sharper analytical tool than can be achieved by pseudo-spectral methods.

\section{Current experimental results}

An example of a promising approach based on chaos theory is shown in Fig. 5. (see [20]). Here we see the time trajectories of the representative state vector in 3-dimensional state-space obtained by the delay-time embedding method using the following equation

$$
\begin{aligned}
& S_{i}=[\mathbf{x}(i), \mathbf{x}(i+L), \ldots, \mathbf{x}(i+(m-1) L)] \\
& \mathbf{S} \in\left(S_{1}, S_{2}, \ldots, S_{n-m}\right)
\end{aligned}
$$

where $L$ is time lag,

$$
\begin{array}{ll}
\mathbf{S} & \text { state vector, } \\
m & \text { state dimension, } \\
\mathbf{x} & \text { data vector, } \\
n & \text { number of data samples. }
\end{array}
$$

Evidently, the form of such trajectories for different levels of proband attention varies significantly. However, in order to generalize such observations into a hypothesis that can later on be verified and used as basis for constructing of some
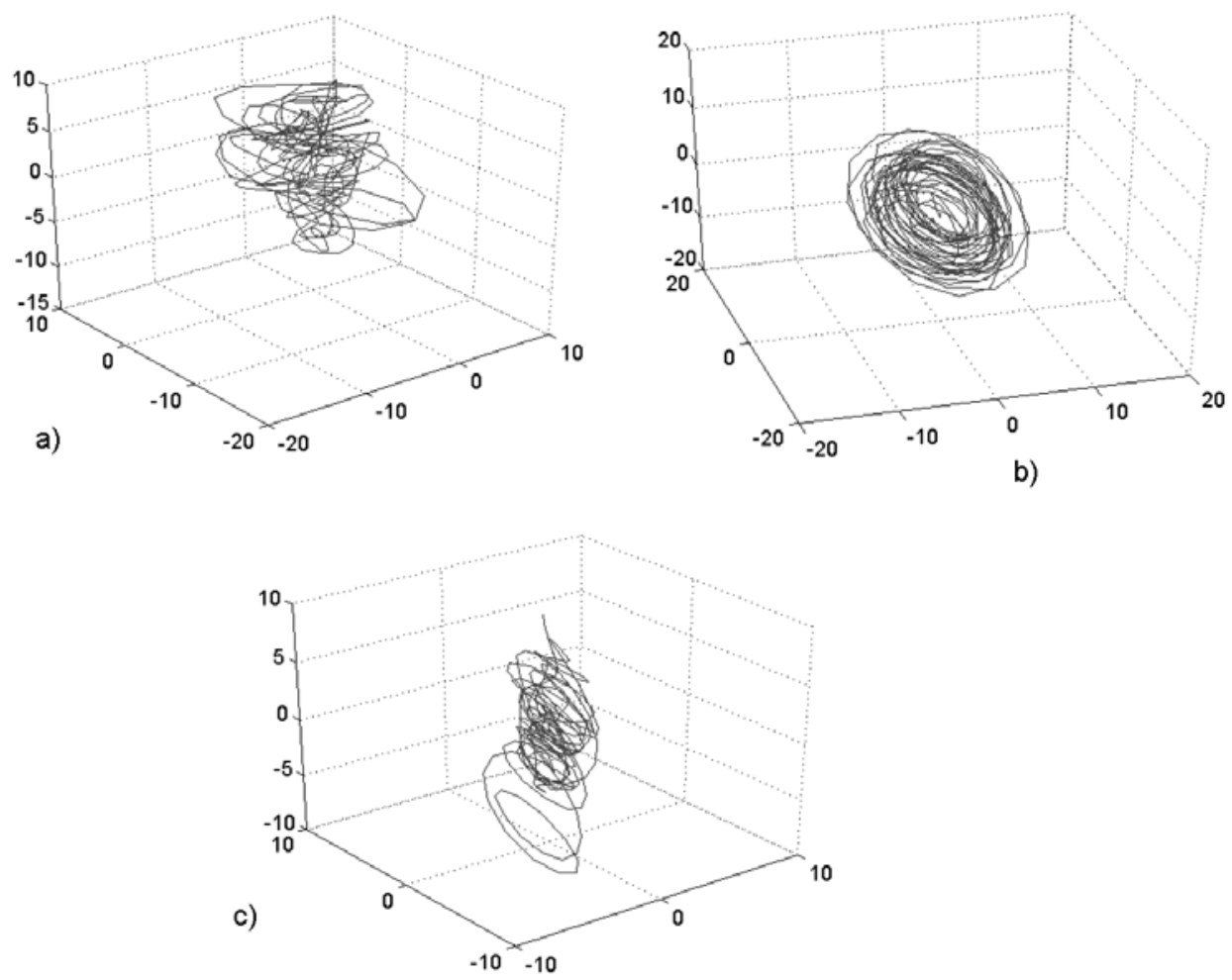

Fig. 5: An example of the "chaotic" state-space trajectory of the proband in the stage of full vigilance (a), somnolence (b) and hypnagogium (c). In all cases 3 second sample are shown. 


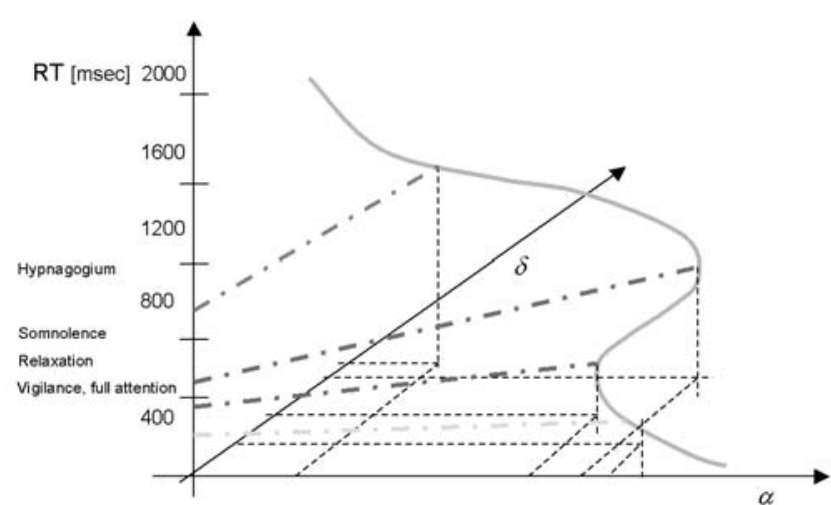

Fig. 6: Example of a typical relation between pseudo-spectral components $\alpha, \delta$ and reaction time RT micro-sleep warning device, we need to have access to many more measurements.

Nevertheless, on the basis of a comparison of the results from analyses already made of the above mentioned pseudo-spectral measurements compared with real time measurements of the particular proband reaction-time RT and the correctness of the subject's response to a stimulus, we have found dependences such as those that shown schematically in Fig. 6.

This relation should be taken as an example only, although it is the result of careful measurement. This is because of the very great individuality of the structure and distribution of EEG signals.

We now have available a set of complete measurements performed on about 30 human subjects. This seems to be enough to formulate the hypothesis that in will be possible to estimate the actual and expected attention level of a given

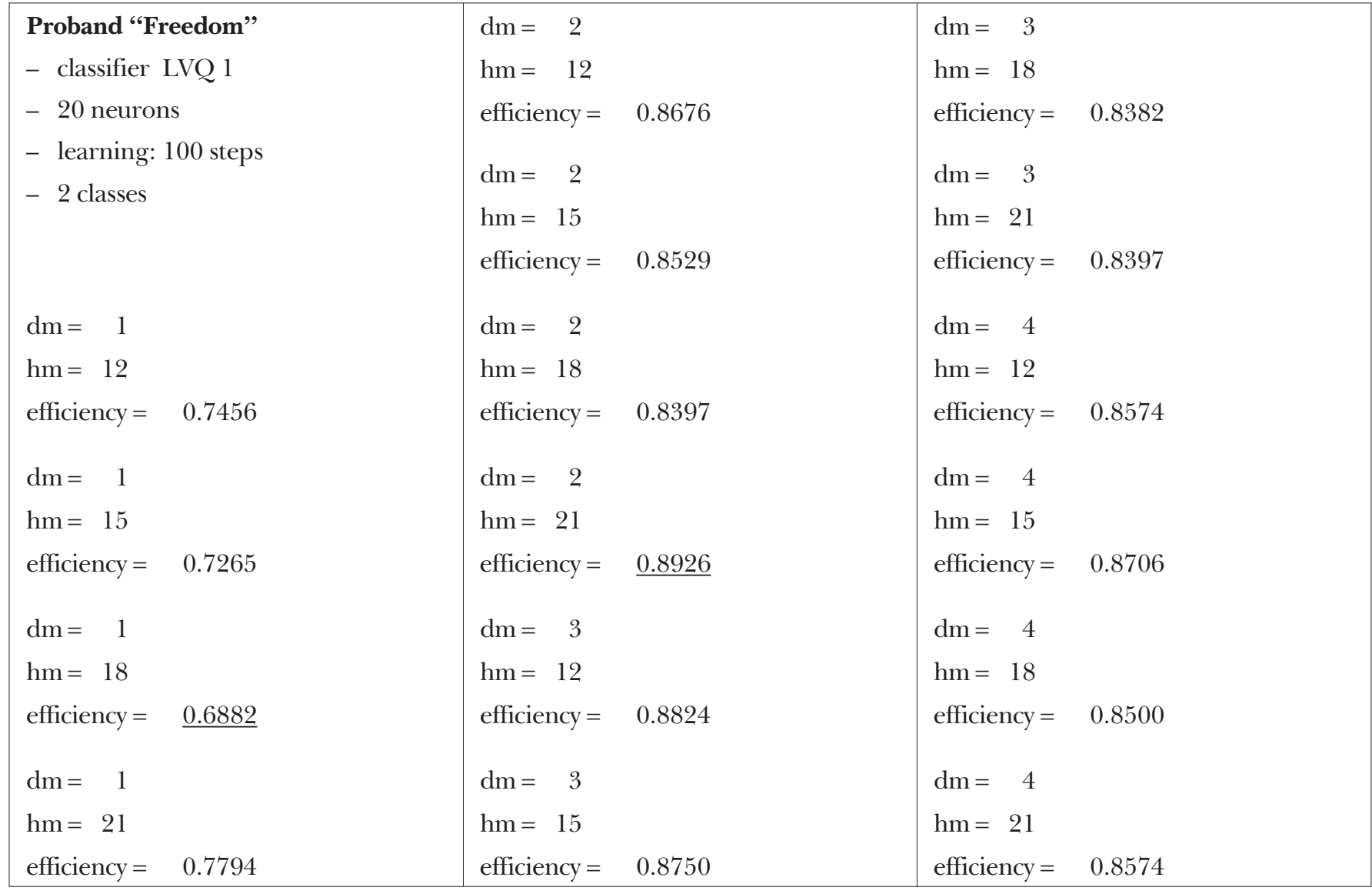

Example of classification results for one sample of data: Freedom, frequency band 3-12Hz

\begin{tabular}{llllllllllllll} 
Obtained result & 2 & 2 & 2 & 2 & 2 & 1 & 1 & 2 & 1 & 2 & 1 & 2 & 1 \\
Correct & 2 & 2 & 2 & 2 & 2 & 1 & 1 & 2 & 1 & 2 & 1 & 2 & 1 \\
\hline Obtained result & 2 & 2 & 2 & 1 & 1 & 1 & 2 & 2 & 2 & 2 & 1 & 1 & 1 \\
Correct & 1 & 2 & 2 & 1 & 1 & 1 & 2 & 2 & 2 & 1 & 1 & 1 & 1 \\
\hline Obtained result & 2 & 2 & 2 & 1 & 2 & 1 & 2 & 2 & 1 & 1 & 2 & 1 & 2 \\
Correct & 2 & 2 & 2 & 1 & 2 & 1 & 1 & 2 & 1 & 1 & 1 & 1 & 2
\end{tabular}




\begin{tabular}{|c|c|c|c|c|c|c|c|c|c|c|c|c|c|c|c|c|}
\hline Obtained result & 2 & 2 & 2 & 1 & 2 & 1 & 1 & 2 & 2 & 2 & 1 & 2 & 1 & & & \\
\hline Correct & 1 & 2 & 1 & 1 & 1 & 1 & 1 & 2 & 2 & 1 & 1 & 2 & 1 & & & \\
\hline Obtained result & 2 & 2 & 2 & 1 & 1 & 2 & 1 & 1 & 2 & 2 & 2 & 2 & 1 & 2 & 2 & 2 \\
\hline Correct & 2 & 2 & 1 & 1 & 1 & 2 & 1 & 1 & 2 & 2 & 1 & 2 & 1 & 2 & 2 & 2 \\
\hline
\end{tabular}

Key: 1 vigilance

2 somnolence

Fig. 7: Example of results obtained by various neural network based micro-sleep classifiers

human subject interacting with an artificial system, on the basis of knowledge of the pseudo-spectral components $\alpha$ and $\delta$, and from a prediction of their values for a satisfactory prediction horizon.

On the basis of such a hypothesis, we attempted to develop a set of micro-sleep classifiers. Here the application of artificial neural networks (especially LQV and RBF networks) gave quite interesting results (see [19] e.g.), an example of which is shown in the tables presented in Fig. 7.

The reliability of such a classification was in this case in the region between 69 to $89 \%$. The level of correspondence between the obtained and correct results for other probands and other types of neural network classifiers that we were used were slightly better (about $90 \%$ ). We can therefore say that this approach seems to be an acceptable starting point for further research in this direction.

\section{Conclusions}

The presently available base of experimental results is too small for the results to be generalized and to verify not only the basic hypothesis, but also the usefulness of these approaches to micro-sleep classification.

Many more measurements on several hundreds or even thousands of subjects will be necessary. Of course, this is beyond the capacity of a single laboratory.

An attempt has therefore been made to stimulate cooperation in this research from other laboratories, institutes, universities and possibly companies in the Czech Republic and abroad.

We hope that this research, representing a significant part of the activity of the newly created Czech National Node for Neuroinformatics, can be included in the long term program of the Global Neuroniformatic Net of the OECD Global Science Forum. In the framework of such a project, a so-called Micro Sleep Base will be developed and results will be collected. Compatible measurements will be made of the EEG activities of many people, supplemented by data on necessary auxiliary measurements such as in-time reaction time, correctness of on-stimulus response etc. The data will be stored, processed, mined and distributed to researchers.

This seems to be the best way to verify the hypothesis and test the usefulness of our approach.

Our work up to now indicates that, though the dependences between the components of EEG pseudo-spectra and reaction time are very individual and differ for each subject, they do not change within the lifetime of an individual subject (except in the event of some serious illnesses or injuries).
This seems to be very significant, because it allows us to put forward the idea that in due course an individually applicable tool for classifying and predicting the attention level of an operator of a specific (namely transportation) system can be developed. This will warn him/her (and also a supervisor) of the imminent possibility of falling into dangerous stages of interaction with the artificial system, and will improve the potential to prevent accidents.

\section{References}

[1] Nilsson, T., Nelson, T. M., Carlson, D.: Development of fatigue symptoms during simulated driving. Accid Anal Prev., July 1997, Vol. 29, No. 4, p. 479-488.

[2] Marottoli, R. A., Richardson, E. D., Stowe, M. H., Miller, E. G., Brass, L. M., Cooney, L. M. Jr, Tinetti, M. E.: Development of a test battery to identify older drivers at risk for self-reported adverse driving events. J. Am. Geriatr. Soc., May 1998, Vol. 46, No. 5, p. 562-568.

[3] Arnold, P. K., Hartley, L. R., Corry, A., Hochstadt, D., Penna, F., Feyer, A. M.: Hours of work, and perceptions of fatigue among truck drivers. Accid Anal Prev., July 1997, Vol. 29, No. 4, p. 471-477.

[4] Novák, M.: Theory of System Tolerances. Czech. Praha: Academia, 1987.

[5] Samel, A., Wegmann, H. M., Vejvoda, M.: Aircrew fatigue in long-haul operations. Accid Anal Prev., July 1997, Vol. 29, No. 4, p. 439-452.

[6] Samel, A., Wegmann, H. H., Vejvoda, M., Wittiber, K.: Stress and fatigue in long distance 2-man cockpit crew. German. Wien Med. Wochenschr., 1996, Vol. 146, No. 13-14, p. 272-276.

[7] Taylor, J. G., Alavi, F. N.: A Global Competitive Network for Attention. Neural Network World, 1993, Vol. 3, No. 5, p. 477-502.

[8] Tzyy-Ping, J., Makeig, S., Stensmo, M., Jejnowski, T. J.: Estimating Alertness from the EEG Power Spectrum. IEEE Transaction on Biomedical Engineering, January 1997, Vol. 44, No. 1, p. 60-69.

[9] Jansen, B. H., Bourne, J. R., Ward, J. W.: Autoregressive Estimation of Short Segment Spectra for Computized EEG Signals. IEEE Transaction on Biomedical Engineering, September 1981, Vol. BME-28, No. 9, p. 630-638.

[10] Makeig, S., Elliot, F., Inlow, M., Kobus, D.: Lapses in Alertness: Brain-evoked responses to task-irrelevant auditory probes. San Diego (CA): Naval Health Research Center, 1992, Tech. Rep., p. 90-93. 
[11] Fruhstorfer, H., Bergstrom, R.: Human vigilance and auditory evoked responses. Electroencephalogr. Clin. Neurophysiol., 1969, Vol. 27, No. 4, p. 346-355.

[12] Makeig, S., Inlow, M.: Lapses in: Alertness Coherence of fluctuations in performance and EEG spectrum. Electroencephalogr. Clin. Neurophysiol., 1993, Vol. 86, p. 23-35.

[13] Matoušek, M., Petersén, I.: A method for assessing alertness fluctuations from EEG spectra. Electroencephalogr. Clin. Neurophysiol., 1983, Vol. 55, No. 1, p. 108-113.

[14] Belyavin, A., Wright, N.: Changes in electrical activity of the brain with vigilance. Electroencephalogr. Clin. Neurophysiol., 1987, Vol. 66, No. 2, p. 137-144.

[15] Venturini, R., Lytton, W., Sejnowski T.: Neural Network Analysis of event related potentials and electroencephalogram. In: Advances in Neural Information Processing Systems, Eds: J. E. Moody, S. J. Hanson, R. P. Lippmann, San Mateo, (CA): Morgan Kaufman, 1992, Vol. 4, p. 651-658.

[16] Makeig, S., Elliott, F., Postal, M.: First demonstration of an alertness monitoring/management system. San Diego (CA): Naval Health Research Center, 1993, Tech. Rep. No. 93-36.

[17] Makeig, S., Jung, T.: Alertness is a principal component of variance in the EEG spectrum. NeuroRep., 1995, Vol. 7, No. 1, p. 213-216.

[18] Cohen, J.: Human Robots in Myth and Science. South Brunswick and New York: A. S. Barnes and Company, 1966.

[19] Tatarinov, V.: Klasifikace bdělosti operátora. (Czech, Classification of operator vigility), Prague: CTU in Prague, Faculty of Transportation Sciences, Laboratory of System Reliability, 2001, Res. Rep. No: LSS 107/01.

[20] Svoboda, P.: Metody analýzy EEG aktivity. (Czech, Methods of EEG Activity Analysis), Diploma thesis, Prague: CTU in Prague, Faculty of Electrical Engineering, 2001.
[21] Svoboda, P.: Alternative Methods of EEG Signal Analysis. Neural Network World, 2002, Vol. 12, No. 3, p. 255-260.

[22] Faber, J., Šrutová, L., Pilařová, M.: EEG Spectrum as Information Carrier. Sborník lék., 1999, Vol. 100, p. 191-204.

[23] Novák, M., Faber, J., Přenosil, V., Valach, I.: Thalamo-cortical Oscillations based Prediction of Micro-Sleeps. Neural Network World, 1999, Vol. 9, No. 6, p. 527-546.

[24] Novák, M., Votruba, Z.: Reliability of man-system interaction and theory of neural networks operations. Siena (Italy): NATO Workshop Limitations and Future Trends in Neural Computing, October 22-24, 2001.

[25] Novák, M.: Artificial systems operation - Problems in safety and reliability. Rethymno (Crete, Greece): Multiconference CSCC, MCP, MCME, July 9, 2001.

Prof. Dr. Mirko Novák, corresponding author phone: +420224359548, +420602242870

fax: +420224359545

e-mail:mirko@fd.cvut.cz

Prof. MUDr. Josef Faber

Prof. Zdeněk Votruba

Dr. Tomáš Tichý

Ing. Petr Svoboda

Ing. Vladimír Tatarinov

Department of Control Engineering and Telematics Laboratory of System Reliability

Czech Technical University in Prague

Faculty of Transportation Sciences

Konviktská 20

11000 Prague 1, Czech Republic

Col. Prof. Václav Přenosil

Military Academy, Brno

Kounicova 65

61200 Brno, Czech Republic 